\title{
Penile Cancer pT4 TNM Finding v7
}

National Cancer Institute

\section{Source}

National Cancer Institute. Penile Cancer pT 4 TNM Finding v7. NCI Thesaurus. Code C89175.

Penile cancer with tumor invading other adjacent structures. (from AJCC 7th Ed.) 